\title{
Comparison and Research with Different Protection Circuit of Chopper Cascade Speed System When Inverter failure
}

\author{
Shiyang Su \\ School of North China Electric Power University,Baoding 071051, China; \\ Sushiyang88@sina.com
}

Keywords: electronic-protection circuit, high-voltage power failure, active inverter, inverter subversion.

\begin{abstract}
Firstly, according to the topology of conventional chopper cascade speed system to analyze the transient process of main loop during of contactor operation when device failure. Because the contactor action requires a longer time, Equipment is vulnerable to the impact of high voltage, high current during the contactor action, and cause damage to power electronic devices. For contactors drawbacks, the main circuit topology with electronic protection circuit is designed. Then analyze transient process of conventional chopper cascade speed system and chopper cascade speed system with electronic protection circuit under high voltage power failure conditions, and theoretics is detailedly deduced. The basis of theoretical analysis under high-voltage power failure condition is get. Finally, simulation model of conventional chopper cascade speed system and with electronic protection circuit is built respectively in Matlab, and research on simulation under high-voltage power failure condition. Simulation results verify the correctness of theoretical analysis and derivation, and show that electronic protection circuits effectively solve the problem of high-voltage power failure under active inverter structure and inverter subversion.
\end{abstract}

\section{Introduction}

In this paper, we first introduce the conventional chopper cascade speed regulation system of main circuit topology, and analyze the transient process of the main circuit process in the contactor protection action. Contactor action time is longer, usually tens of milliseconds to $1200 \mathrm{~ms}$, and the movement phase speed control equipment is susceptible to high voltage, large current impact, resulting in damage to the equipment of speed control, and showing that the contactor can not be effectively control the equipment fault protection. Concerning that the contactor on-off time is long, the electronic protection circuit is designed based on the conventional chopper control system structure. Then we analyze with the high pressure loss of electrical conditions, the transient process of the conventional chopper speed control system and the electronic protective circuit of the chopper cascade speed regulation system. Meanwhile the detailed theoretical derivation is done, which can be an evidence that the high pressure cause the electrical loss. Finally, the simulation model of conventional chopper on speed regulation system and electronic protective circuit of the chopper cascade speed regulation system are built in Matlab. And the simulation research on high voltage power failure conditions is done. The results are basically in accordance with the theoretical analysis, which indicate that the electronic protective circuit is effective in solving the problem of the active inverter structure under high pressure loss of electricity or inverter subversion problem (pulse loss, thyristor interdiction ability weak) ${ }^{[3-5]}$.

\section{Electronic Protection Circuit Principle and Structure}

At present, if there is something wrong with the chopper cascade speed regulation system, we generally rely on contact points and action for protection. Using contactor to action protection has the following advantages:

(1) the contactor control movement is simple, not like power electronic devices which need extra drive circuit; 
(2) when the contactor is under high voltage, the ability of large current shock is stronger than e power lectronics device;

In view of the above advantages, the chopper cascade speed regulation system of regular used more action to protect contactor, figure 1 is conventional chopper cascade speed regulation system structure diagram. It can be seen from the figure 1.

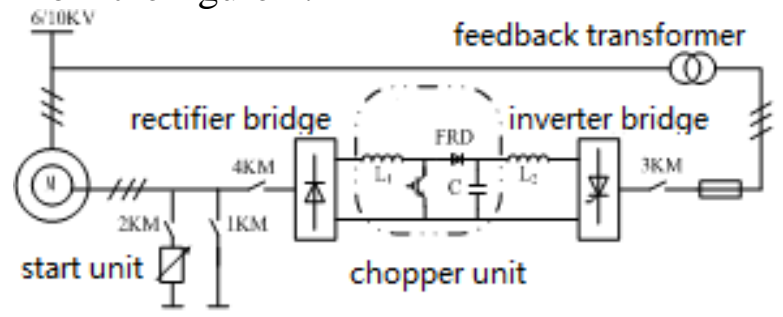

Figure.1

The regular chopper cascade speed system use contact points and action for protection when failed in conventional control, the time of the contactor is generally a couple of and one or two milliseconds, even with a few milliseconds action for a long time, during its movement speed regulation system is impacted by the high voltage, high current, it may damage the power electronic devices. In view of the contactor action's long time, it is difficult to protect the instant fault. Through researching the regular chopping cascade speed regulation system in-depth, chopper cascade speed regulation system was proposed with electronic protection circuit. Electronic protection using thyristor as protection device in the circuit, the switching time of the thyristor is the microsecond, much less than the action time of the contactor. Figure 2 is the primary loop structure of chopper cascade speed regulation system with electronic protection circuit.

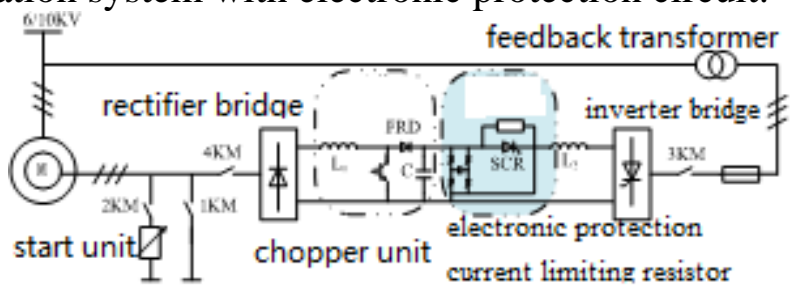

Figure.2

It can be seen from the figure2, comparing with figure1, increase structure electronic protection circuit in the primary loop, increase thyristor SCR protection namely between inverse diode FRD resistance and protection of thyristor parallel current limiting resistor R1, and protect the thyristor turn-off reverse bias circuit. When the chopper cascade speed regulation system is at normal speed, protect the thyristor conduction, short answer the current limiting resistor R1; When malfunction chopper cascade speed regulation system failed, sent out protection circuit signal which means turning off thyristor protection, at the same time sent out signal contactor action, before the contactor action, protect the thyristor turn-off, the jumper current-limiting resistor R1 series in the primary loop of the inverter dc current loop current to limit the rise ${ }^{[6]}$.

The main loop resistance of general chopper cascade speed regulation system is small, which can be ignored; the speed regulation with electronic protection circuit of chopper cascade speed regulation system was failed, current limiting resistor R1 that series changed inverter side of the circuit topology, by regulating normal owe attenuation circuit into a damped oscillation damping of oscillation circuit or owe damped oscillation damping circuit of inverter dc current limiting the rise, which limit current in the inverter bridge thyristor bear range, so that avoid a hit from the large current inverter bridge, achieving the purpose of protection of thyristor inverter bridge.

\section{Analysis of High Voltage Power Loss after the Electronic Protection Circuit}

Due to the long operation time of the contactor, and speed regulating main circuit fault current will keep upper during this period, value exceeds the tolerance of thyristor, which can cause device damage, and suggest that the contactor can not effectively protect speed control equipment fault. In view of the drawbacks of the contactor protection, electronic protection of main circuit topology is 
designed.

\subsection{Analysis on the Protection of the Contactor after Hhigh Voltage Power Loss}

Chopper cascade speed regulation system adds the equivalent series circuit DC potential by changing the accounting for cycle, while after the high pressure electricity loss, the equivalent DC EMF suddenly lose. Due to the resistance of the loop is very small, only the line resistance, the inverter can be simplified as a RLC circuit. At this time, the inverter DC current rise quickly and the amplitude will very high ${ }^{[7]}$. Figure 3 for chopper speed control system for normal operation and high voltage loss of power inverter equivalent circuit.

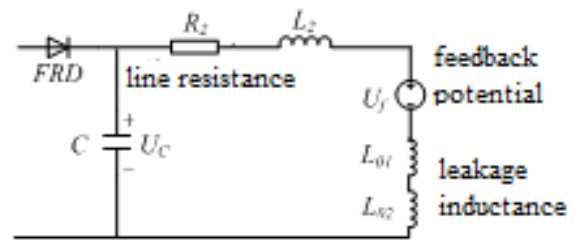

Figure.3

(a) the Equivalent Circuit Structure of the Inverter Side during Normal Operation

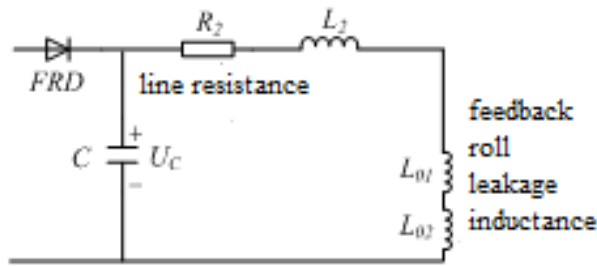

(b) the Equivalent Circuit Structure of the Inverter side when the high voltage is lost

According to the circuit of Figure3(b), the KVL equation of high voltage power loss is established: $L C \frac{d^{2} u_{c}}{d t^{2}}+R_{2} C \frac{d u_{c}}{d t}+u_{c}=0$

In this formula, $\mathrm{C}$ is the capacitance value, the UC is the capacitance voltage, the R2 is the line circuit, and the $\mathrm{L}$ is the total inductance of the circuit.

Solving the Formula (1), the characteristic root can be gained: $p=-\frac{R_{2}}{2 L} \pm \sqrt{\left(\frac{R_{2}}{2 L}\right)^{2}-\frac{1}{L C}}$

For chopper cascade speed control device, the circuit resistance of the main circuit is small:

$\left(\frac{R_{2}}{2 L}\right)^{2}-\frac{1}{L C}<0$

The Formula (3) shows that the Formula (1) is provided with a pair of conjugate roots. Supposing that: $\delta=R_{2} / 2 L, \omega^{2}=1 / L C-\left(R_{2} / 2 L\right)^{2} ; \quad$ a characteristic root is available: $\left\{\begin{array}{l}p_{1}=-\delta+j \omega \\ p_{2}=-\delta-j \omega\end{array}\right.$ (4)

For the sudden high voltage power loss, assuming the $t=0$ time, the initial condition in the circuit is the formula (4), that is, the high voltage power loss instantaneous capacitor voltage value $U_{0}$ and the current value of the flow circuit $\quad I_{0}:\left\{\begin{array}{l}u_{c(0-)}=u_{c(0+)}=U_{0} \\ i_{(0-)}=i_{(0+)}=I_{0}\end{array}\right.$

Then the capacitor voltage $u_{c}$ is as follows:

$$
u_{c}=A_{1} e^{p_{1} t}+A_{2} e^{p_{2} t}
$$

$$
A_{1}=\frac{I_{0}+U_{0} p_{2} C}{C\left(p_{2}-p_{1}\right)} 、 A_{2}=-\frac{I_{0}+U_{0} p_{1} C}{C\left(p_{2}-p_{1}\right)}
$$

Finally, the capacitor voltage $u_{c}$ can be derived:

$$
\begin{aligned}
u_{c} & =\frac{I_{0}+U_{0} p_{2} C}{C\left(p_{2}-p_{1}\right)} e^{p_{1} t}-\frac{I_{0}+U_{0} p_{1} C}{C\left(p_{2}-p_{1}\right)} e^{p_{2} t} \\
& =-\frac{I_{0}}{\omega C} e^{-\delta t} \bullet \sin \omega t+\frac{U_{0} \omega_{0}}{\omega} e^{-\delta t} \bullet \sin (\omega t+\beta)
\end{aligned}
$$

During the formula: $\quad \omega_{0}=\sqrt{\delta^{2}+\omega^{2}}, \quad \beta=\operatorname{arctg}(\omega / \delta)$ 。

By the Formula (8) derivation, current through the inverter can be derived, which is DC current 
loop:

$$
\begin{aligned}
i & =-C \frac{d u_{c}}{d t} \\
& =-\frac{I_{0} \omega_{0}}{\omega} e^{-\delta t} \sin (\omega t-\beta)+\frac{U_{0}}{\omega L} e^{-\delta t} \sin \omega t
\end{aligned}
$$

By the Formula (9) derivation, the time to reach the maximum value of high voltage power loss can be deduced.

$$
\begin{aligned}
\frac{d i}{d t}=0 & \Rightarrow \frac{d\left(-\frac{I_{0} \omega_{0}}{\omega} e^{-\delta t} \sin (\omega t-\beta)+\frac{U_{0}}{\omega L} e^{-\delta t} \sin \omega t\right)}{d t}=0 \\
& \Rightarrow \operatorname{tg} \omega t=\frac{\frac{U_{0}}{L}-I_{0} \omega \cos \beta-I_{0} \omega \delta \sin \beta}{\frac{U_{0} \delta}{L}+I_{0} \omega \sin \beta-I_{0} \omega \delta \cos \beta} \\
& \Rightarrow t_{\text {Max }}=\frac{\operatorname{arctg}\left(\frac{U_{0}}{\frac{U_{0} \delta}{L}-I_{0} \omega \cos \beta-I_{0} \omega \sin \beta-I_{0} \omega \delta \cos \beta}\right)}{\omega} \\
I_{\text {Max }}=-\frac{I_{0} \omega_{0}}{\omega} e^{-\delta t_{\text {Max }}} \sin \left(\omega t_{\text {Max }}-\beta\right) & \\
& +\frac{U_{0}}{\omega L} e^{-\delta t_{\text {Max }}} \sin \omega t_{\text {Max }}
\end{aligned}
$$

\subsection{Analysis of the Electronic Protection Circuit of the Speed Regulating System after the High Voltage Power Loss}

After the chopper reverse blocking diode FRD and reactor between the L2 increased protection thyristor SCR, also in the protection of the crystal at both ends of the cross connecting an appropriate resistance resistance. Because the protection of the crystal thyratron needs to reverse bias voltage, the circuit increase anti bias circuit. The protection of thyristor tube in the normal speed is in a conduction state, meanwhile high voltage loss of power (speed regulating equipment failure) is off, the two ends of the resistance will be in series circuit, limit current and consume the energy stored in the capacitor ${ }^{[8]}$. Chopper cascade speed regulation system with electronic protective circuit of inverter equivalent circuit as shown in Figure 4 below.

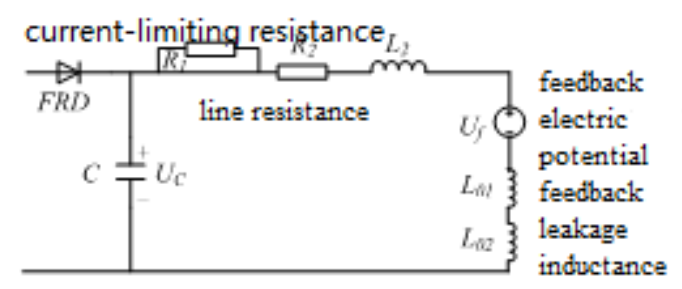

Figure4

(a) the Equivalent Circuit Diagram of the Inverter side of the chopper cascade speed regulation with electronic protection

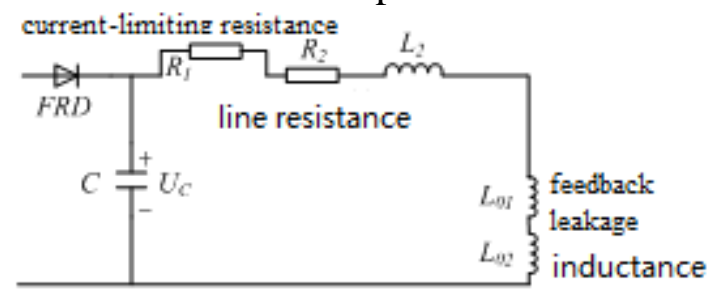

(b) the Equivalent Circuit Diagram of the Inverter side of the chopping speed regulating system with electronic protection

Figure 4 (b) shows that, when the high voltage loses electrical (control system fault) loop 
resistance R: $R=R_{1}+R_{2}$. Inverter can be equivalent to RLC circuit ${ }^{[9-10]}$. After loop in series into a current limiting resistor becomes non-oscillatory discharge or excessive damping discharge process can be called. At that time, it is necessary to meet the following conditions: $R>2 \sqrt{\frac{L}{C}}$

$$
\begin{aligned}
u_{c}= & \frac{I_{0}+U_{0} p_{2} C}{C\left(p_{2}-p_{1}\right)} e^{p_{1} t}-\frac{I_{0}+U_{0} p_{1} C}{C\left(p_{2}-p_{1}\right)} e^{p_{2} t} \\
= & \frac{I_{0}}{C\left(p_{2}-p_{1}\right)}\left(e^{p_{1} t}-e^{p_{2} t}\right) \\
& +\frac{U_{0}}{p_{2}-p_{1}}\left(p_{2} \bullet e^{p_{1} t}-p_{1} \bullet e^{p_{2} t}\right)
\end{aligned}
$$

In the formula, the $U_{0}$ is a high voltage power loss instantaneous capacitor voltage value; $\mathrm{I}_{0}$ is the current value of the high voltage power loss instantaneous flow circuit; the $p_{1}$ and $p_{2}$ are the characteristic roots: $\quad p=-\frac{R}{2 L}+\sqrt{\left(\frac{R}{2 L}\right)-\frac{1}{L C}}, \quad p_{2}=-\frac{R}{2 L}-\sqrt{\left(\frac{R}{2 L}\right)^{2}-\frac{1}{L C}}$

By the Formula (13) derivation, we can derive:

$$
\begin{aligned}
& \frac{d i}{d t}=0 \Rightarrow e^{\left(p_{1}-p_{2}\right) t}=\frac{I_{0} p_{2}^{2}+\frac{U_{0}}{L} p_{2}}{I_{0} p_{1}^{2}+\frac{U_{0}}{L} p_{1}} \\
& \Rightarrow t_{\text {Max }}=\frac{\ln \left(\frac{I_{0} p_{2}^{2}+\frac{U_{0}}{L} p_{2}}{I_{0} p_{1}^{2}+\frac{U_{0}}{L} p_{1}}\right)}{p_{1}-p_{2}}
\end{aligned}
$$

Similarly, $t_{\text {Max }}$ will be drawn into Formula (14), we can derive the maximum current of $I_{\text {Max }}$ :

$$
\begin{aligned}
I_{\text {Max }} & =-\frac{I_{0}}{\left(p_{2}-p_{1}\right)}\left(p_{1} \bullet e^{p_{1} t_{\text {Max }}}-p_{2} \bullet e^{p_{2} t_{\text {Max }}}\right) \\
& -\frac{U_{0}}{L\left(p_{2}-p_{1}\right)}\left(e^{p_{1} t_{\text {Max }}}-e^{p_{2} t_{\text {Max }}}\right)
\end{aligned}
$$

\section{Conclusion}

In view of the drawbacks of the contactor protection, we designed main circuit topology structure with electronic protective circuit. We compared the transient process of conventional chopper cascade speed control system with the one with electronic protective circuit and conducted detailed theoretical deduction and obtained the basis of theoretical analysis in higher voltage power loss. Finally in MATLAB model we built simulation models of conventional chopper cascade speed control system and the one with electronic protective circuit and conducted simulation studies with the focus of high voltage power loss. Our finding was basically consistent with theoretical analysis and deduction. Our finding confirmed that the electronic protection circuit can effectively solve the problem of high voltage power loss or inverter subversion under active inverter structure.

\section{References}

[1].SHANG Debin, LIU Huawei,LIU Gu-lan,et al. Application and development trend of SEC highfrequency chopper cascade governing technique[J]. Electric Switchgear, 2007, (6): 50-53.

[2].MA Yongguang, ZHANG Xiaodong, WANG Bingshu,et al. Dynamic model and simulation of cascade motor[J]. Electric Machines \& Control Application, 2007, 31(4): 11-14.

[3].Wang Shaoyong,Zhang Fanghua. Zero-Crossing Distortion in Single-Phase Boost PFC Converters Using Average Current Control[J]. Transactions of China Electro technical Sosiety, 2011, 26(2): 129-133. 
[4].ZHANG Junwei,WANG Bingshu, JIANG Ping. System modeling and double closed-loop control simulation in chopped wave cascade speed control system[J]. Control Engineering of China, 2011, 18(6): 977-981. 